\title{
DESIGNING INTELLIGENT SYSTEM FOR PLANNING ROBOT MANIPULATOR REAL-TIME MOVEMENT IN UNKNOWN DYNAMIC ENVIRONMENT
}

\author{
Daiva Nemeiksyte, Vitalijs Osadcuks \\ Latvia University of Life Sciences and Technologies, Latvia \\ nemeiksyte.daiva@gmail.com, vtl@tvnet.lv
}

\begin{abstract}
Uncertainty caused by presence of unknown static and dynamic obstacles in the robot's operating area while detecting biological objects and expressed by obstacle detection sensor error level, as well as alternating locations of such obstacles result in the need to develop methods of planning and controlling robot movement. Essence of these methods: after a robot is given a task during the process of planning, a safe trajectory of movement is designed in real time considering the possible robot configurations, as well as information concerning the environment that was detected by the sensors. The desired robot configurations are then formed during the process when the trajectory is generated, that is, time functions. The obtained trajectory is the key control factor that shapes appropriate signals to generate torque, which ensures that the robot moves along a safe trajectory with the least possible error to reach the destination point. Therefore, the subject of research in this article is a robotic arm intended for detection and elimination-destruction of biological objects, and the goal is to develop a method of designing an intelligent system for planning robot manipulator real-time movement in an unknown dynamic environment and perform computer simulation with the obtained system in the V-REP and MatLAB environments. Based on the above, the missing parameters for the experimental mobile robot have been calculated in the article and a model of a robotic arm has been developed to analyse the peculiarities and problems of using an intelligent planning system. As a result of the research performed in the article, significant data have been obtained, based on which a method of planning mobile robot movement in an unknown dynamic environment using an intelligent planning system was proposed allowing to improve the accuracy and efficiency of movement planning.
\end{abstract}

Keywords: dynamic environment, intelligent planning system, neural network, fuzzy logic, robot manipulator.

\section{Introduction}

Planning the movement of a robot, which involves identifying the working route of its grip limited by the task of work and the robot in question, is the major issue in the functioning of autonomous robotic systems and one of the most researchable areas of modern science and practice. Dealing with this problem involves issues related to scientific fields, such as artificial intelligence, algebraic geometry, computer perception, and theory of automatic control [1]. Automation of the movement planning process, minimising the time of preparation and execution and accelerating the switching of a robot from one production task to another, justifies the organisation of flexible production [1-3].

During movement, the robotic grip moves from the starting position and orientation towards the specified position and orientation. In the Cartesian coordinate system, description of the movement points is usually based on the use of a homogeneous transformation matrix. The forward movement of the manipulator is described in the form of a curve, which indicates the movement of the centre of the robot from the start to the target point The curve indicating the orientation of the grip forms the rotational path of the robot. In turn, the manipulator's movement trajectory consists of a set of points that describe the robot's forward movement path and the rotational path of the grip as a function of time [4-6].

The purpose of the manipulator's movement planning is to transform the technical conditions of a particular task into the desired trajectory of the robot when the manipulator moves along the planned path following the control factors.

\section{Materials and methods}

A four-stage method has been developed for designing an intelligent system for planning robot manipulator real-time movement in an unknown dynamic environment (Fig. 1, six-joint robot manipulator was taken as an example). This method is different from the previously analysed one in that it calculates during the stage two, the value of change in the final distance $\left(\Delta d_{n o}\right)$ : $\Delta d_{n o}(i+1)-d_{n o}(i)$. The third input parameter is added as a deliverable to the two inputs of the fuzzy block number-two (FB2) in the stage four $\Delta d_{n o}$. 


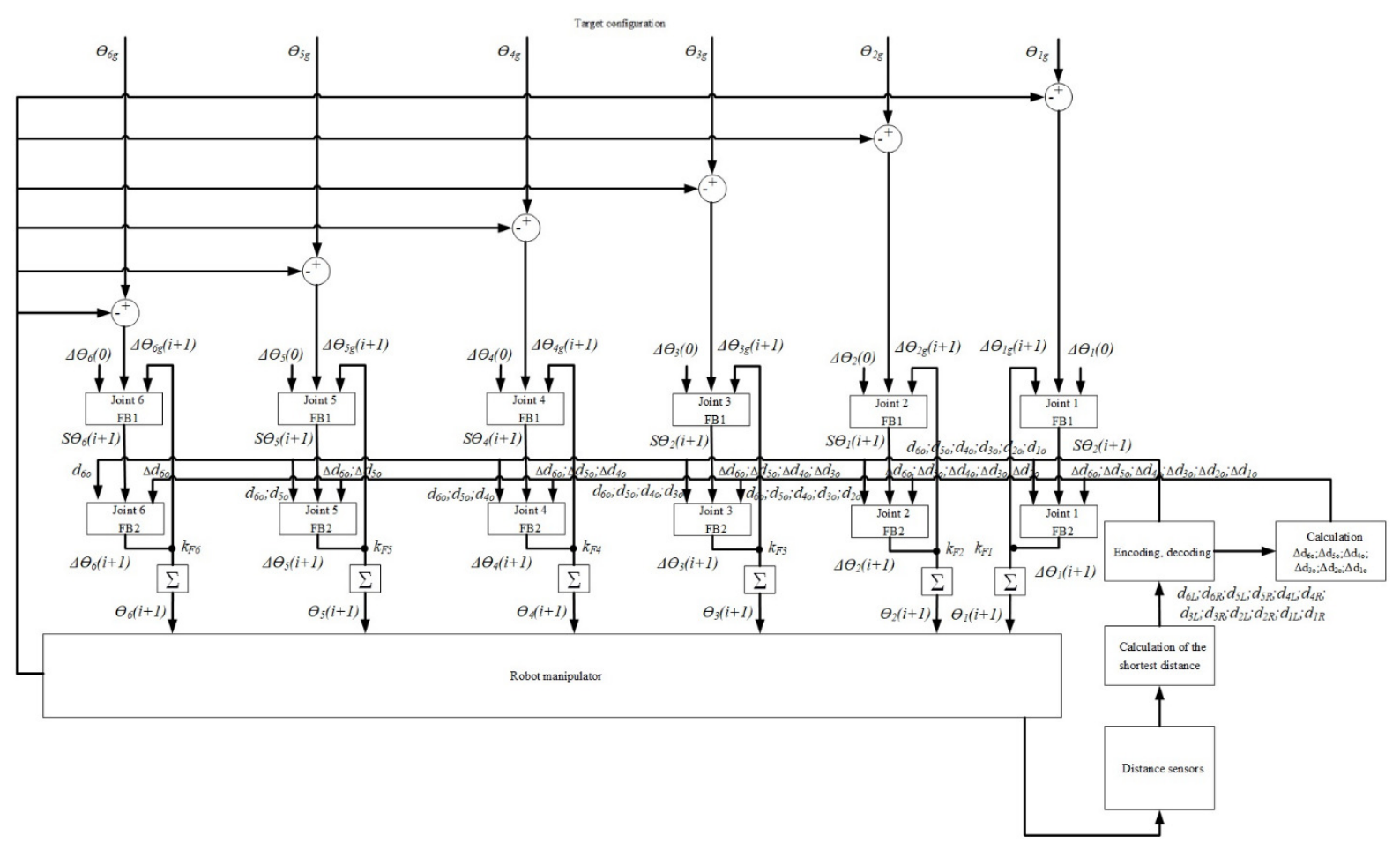

Fig. 1. Intelligent planning system for six joint robot manipulator real-time movement in an unknown dynamic environment

To address the problem of six-joint robot manipulator real-time movement in an unknown dynamic environment an intelligent planning system presented in Fig. 1 was designed. The intelligent planning system for manipulator's real-time movement in an unknown dynamic environment (Fig. 1) present structure involves the unit for calculating the distance obtained after classification. Inputs of this unit are $d_{1 o}, d_{2 o}, d_{3 o}, d_{4 o}, d_{5 o}$ and $d_{6 o}$ outputs are $\Delta d_{1 o}, \Delta d_{2 o}, \Delta d_{3 o}, \Delta d_{4 o}, \Delta d_{5 o}$ and $\Delta d_{6 o}$ [7-10]:

$$
\begin{aligned}
& \Delta d_{1 o}(i+1)=d_{1 o}(i+1)-d_{1 o}(i) ; \\
& \Delta d_{2 o}(i+1)=d_{2 o}(i+1)-d_{2 o}(i) ; \\
& \Delta d_{3 o}(i+1)=d_{3 o}(i+1)-d_{3 o}(i) ; \\
& \Delta d_{4 o}(i+1)=d_{4 o}(i+1)-d_{4 o}(i) ; \\
& \Delta d_{5 o}(i+1)=d_{5 o}(i+1)-d_{5 o}(i) ; \\
& \Delta d_{6 o}(i+1)=d_{6 o}(i+1)-d_{6 o}(i) .
\end{aligned}
$$

The named parameters together with $S \theta_{1}(i+1), S \theta_{2}(i+1), S \theta_{3}(i+1), S \theta_{4}(i+1), S \theta_{5}(i+1)$ and $S \theta_{6}(i+1)$ are used in the stage two of the system as inputs to FB2. The similarity of the two intelligent planning systems for six-joint robot-manipulator movement lays in the same architecture of the possible situation classifications of the movement in an unknown environment based on the designed neural network. It must be noted that the value of $n$-joint parameter $\Delta d_{n o}$ is required to determine the relative velocity at which robot joints approach the moving obstacles, and to determine the dynamics of the unknown objects located in the operating zone. At the same time, based on the graphic analysis of manipulator's operating zone and depending on the direction of the robot joint movement, the value of change in the distance can be both positive and negative.

Designing a fuzzy block two of the n-joint. The structure of the fuzzy planning system of the robot manipulator movement, which is part of the intelligent system under consideration, has the following properties. Firstly, the architecture of its stage one (FB1 of n-joint) is similar to the fuzzy system with the original structures designed for the stage two fuzzy blocks of this intelligent system. Also, important are the coefficients $k_{F 1}$ and $k_{F 2}$ equal to one, because under the conditions when unknown moving obstacles are located in the manipulator's operating zone, to avoid collision with the obstacles, the robot needs to make maximum movement during each software iteration. 
Dependence functions parameters that describe three $n$-joint FB2 inputs $\left(d_{n o}, S \theta_{n}(i+1), \Delta d_{n o}\right)$ and one output $\left(\Delta \theta_{n}(i+1)\right)$, are presented in Fig. 2 , where $d_{n \max }$ is the largest value $d_{n o}$ of change. The following concepts have also been introduced: NL - negative large (change in distance), NM negative medium, NS - negative small, PS - positive small, PM - positive medium, PL - positive large.
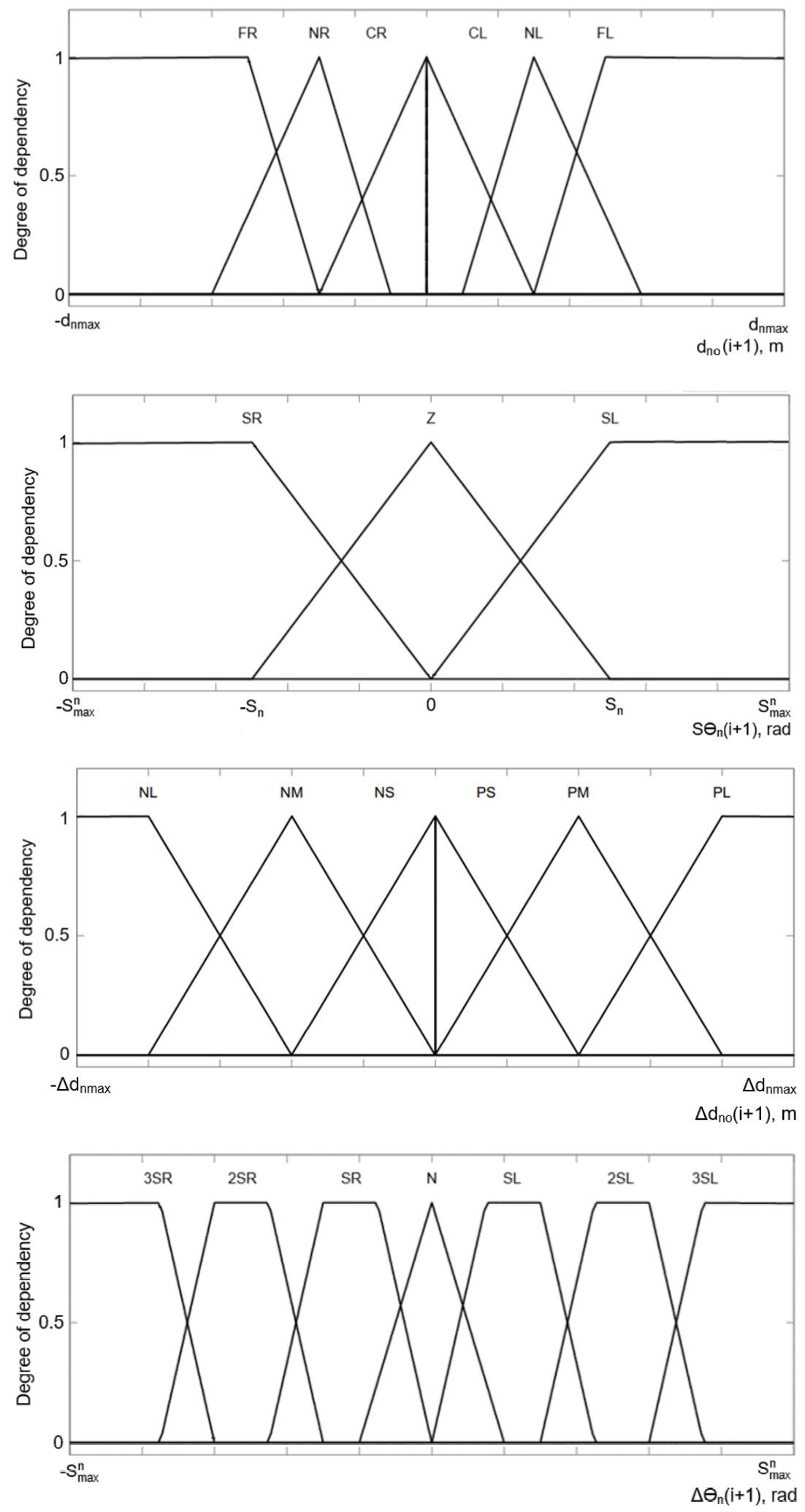

Fig. 2. Dependence functions of FB2 inputs and outputs of a robot manipulator's $n$-joint 
The system of fuzzy basic rules functioning in each FB2 of the n-joint are presented in Table 1.

Fuzzy basic rules functioning in FB2 of robot manipulator $\boldsymbol{n}$-joint

Table 1

\begin{tabular}{|c|c|c|c|c|c|}
\hline No. & $\boldsymbol{d}_{\boldsymbol{n}}$ & $\boldsymbol{S \boldsymbol { \theta } _ { \boldsymbol { n } }}$ & $\boldsymbol{\Delta d}_{\boldsymbol{n} \boldsymbol{0}}$ & $\boldsymbol{\Delta} \boldsymbol{\theta}_{\mathbf{1}}$ & $\boldsymbol{\Delta} \boldsymbol{\theta}_{\mathbf{2}}$ \\
\hline 1 & FR & SR & NL & 3SR & 3SR \\
\hline 2 & FR & SR & NM & 3SR & 3SR \\
\hline 3 & FR & SR & NS & 2SR & 2SR \\
\hline 4 & FR & SR & PS & SR & SR \\
\hline 5 & FR & SR & PM & Z & Z \\
\hline$\ldots$ & $\ldots$ & $\ldots$ & $\ldots$ & $\ldots$ & $\ldots$ \\
\hline 104 & FL & SL & NM & Z & Z \\
\hline 105 & FL & SL & NS & SL & SL \\
\hline 106 & FL & SL & PS & 2SL & 2SL \\
\hline 107 & FL & SL & PM & 3SL & 3SL \\
\hline 108 & FL & SL & PL & 3SL & 3SL \\
\hline
\end{tabular}

\section{Results and discussion}

Computer simulation of an intelligent system of robot manipulator real-time movement planning in an unknown dynamic environment consisted of two tests.

The first test involved placing two moving obstacles (white circles) and two static obstacles (grey circles) in the operating zone (Fig. 3). The first moving obstacle was moving in the direction of the horizontal axis, while the second was moving in the direction of the vertical axis. During the test number-two, there was a single moving vertically unknown obstacle, and three static unknown obstacles (Fig. 7).

During the process of the first test, the manipulator was moving from starting configuration (point A) to target configuration (point B). If $k_{F 1}=1$ and $k_{F 2}=1$, its movement lasted 2119 software iterations performed in $3.427 \mathrm{~s}$ (Fig. 3). The obstacle movement step was equal to $0.036 \mathrm{~m}$ in each iteration. Eventually, the manipulator reached the target point with zero planning error.

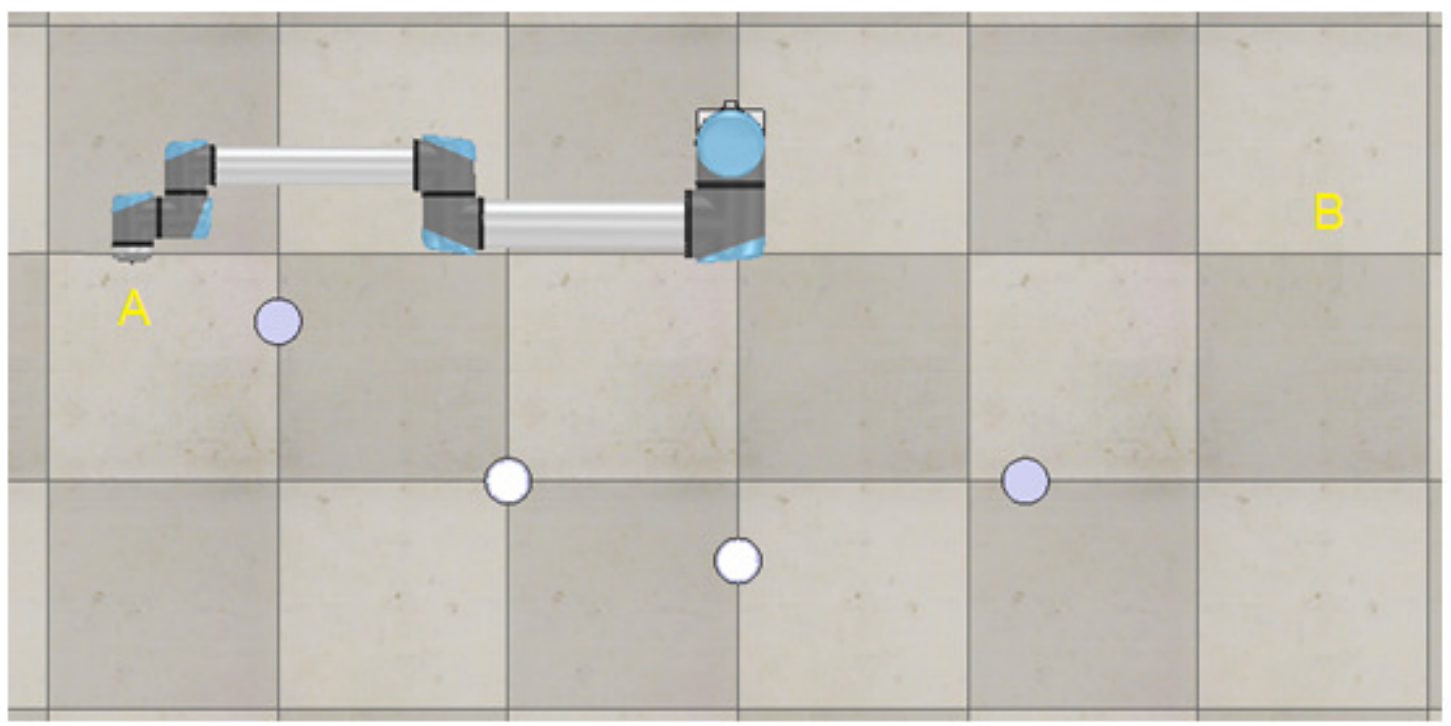

Fig. 3. Results of the test number-one of the intelligent system for planning robot manipulator's real-time movement in an unknown dynamic environment

Figures 4, 5 and 6 present the diagrams of the number-one analysed computer test parameters. 

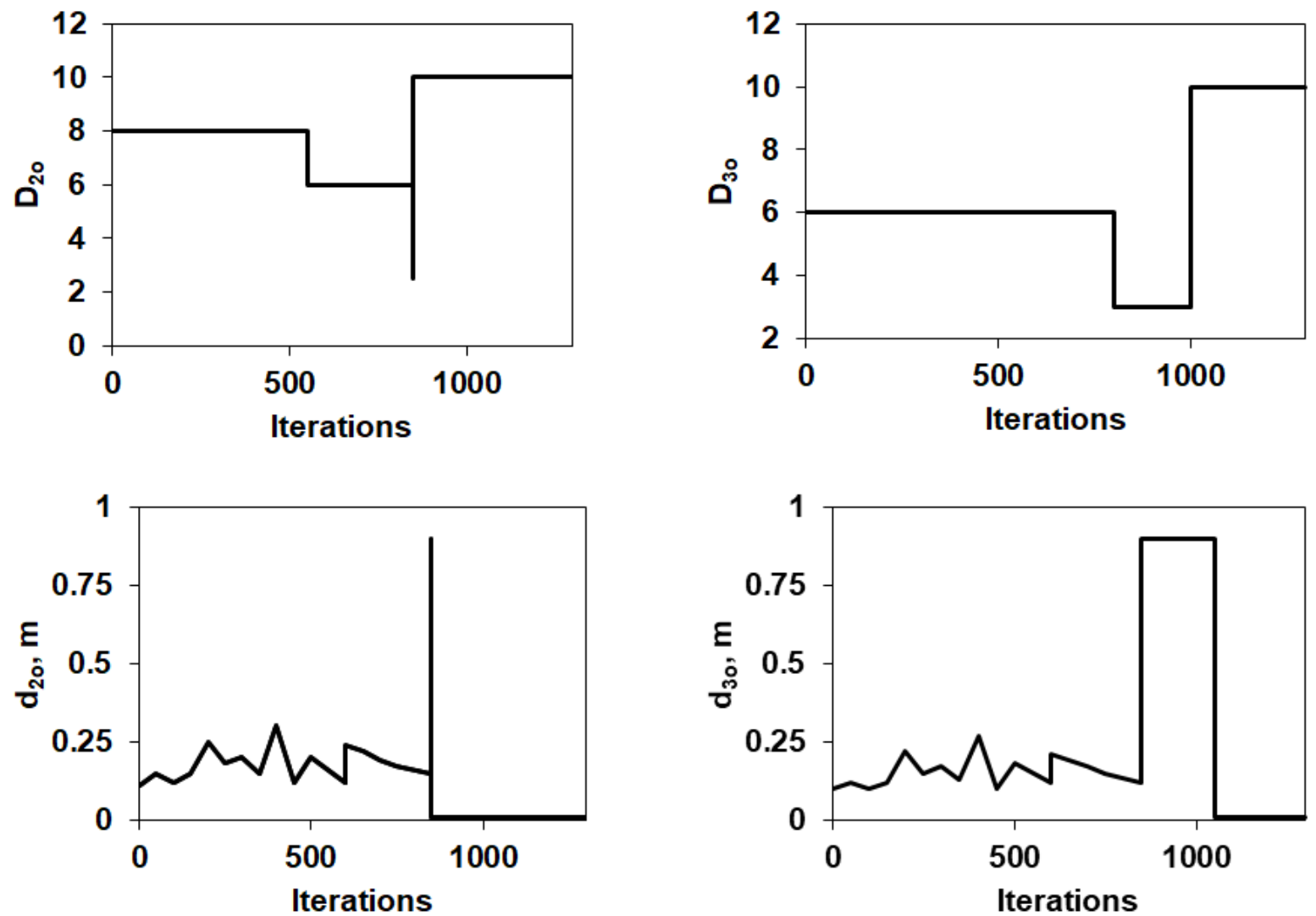

Fig. 4. Test number-one result parameters
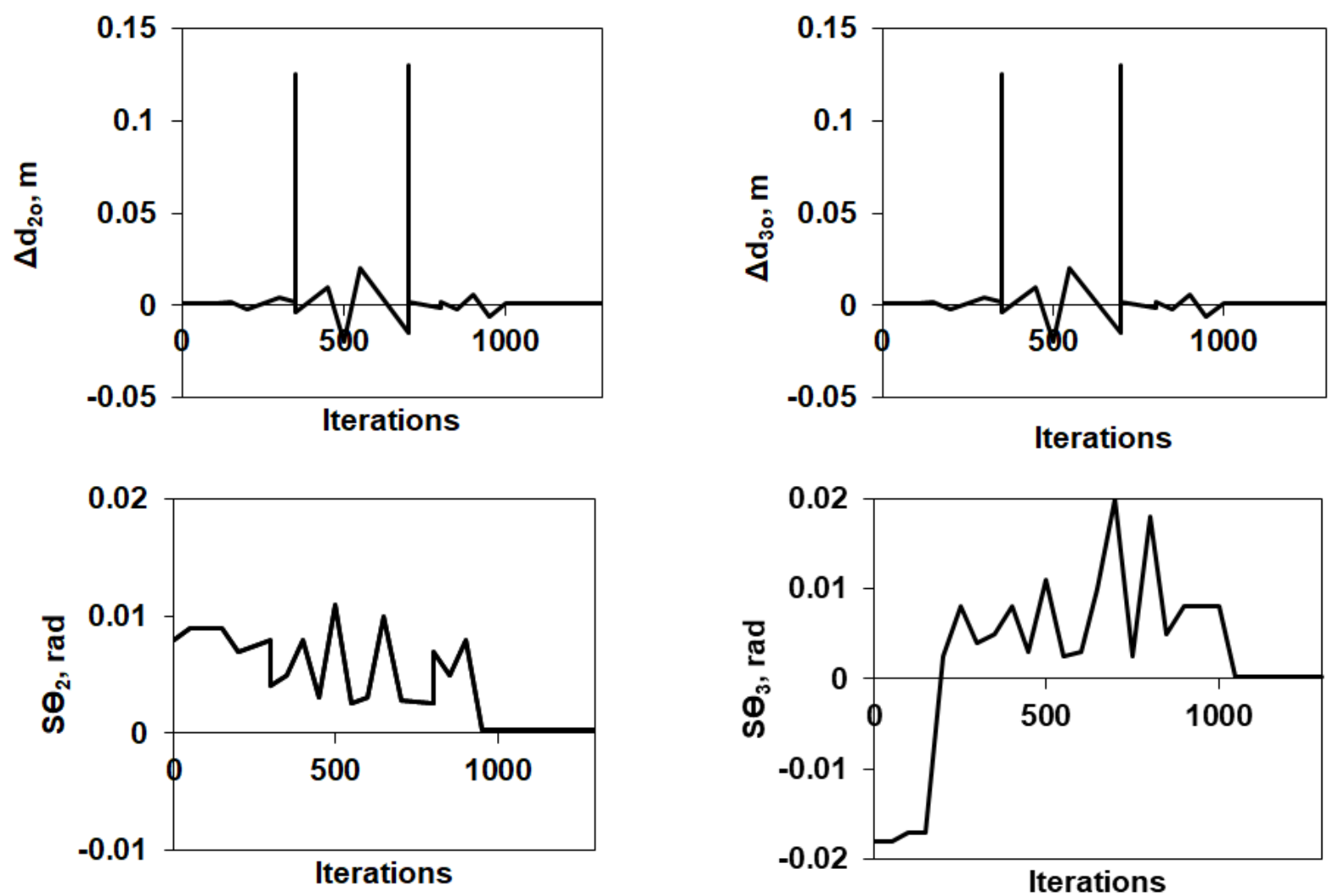

Fig. 5. Test number-one result parameters 

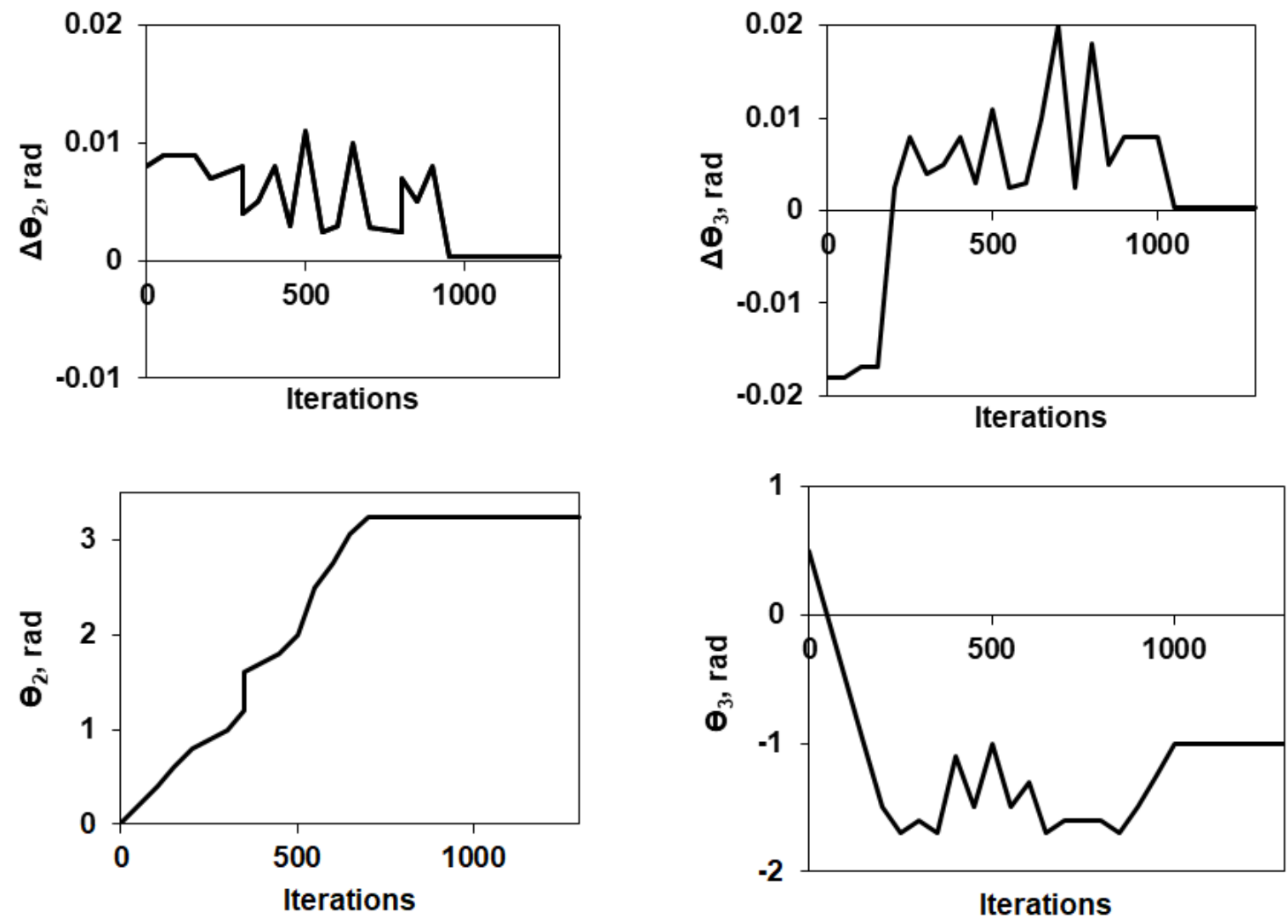

Fig. 6. Test number-one result parameters

During the test number-two, the robot was moving from starting configuration (point A) to target configuration (point B) during 2013 software iterations that lasted $3.7 \mathrm{~s}\left(k_{F 1}=1\right.$ and $\left.k_{F 2}=1\right)$ (Fig. 7). The only moving obstacle (white circles) was moving during each software iteration by a step equal to $0.036 \mathrm{~m}$. Thus, $\Theta_{3}$ planning error was equal to zero, and there were no error of $\Theta_{2}$, because the robot's joint number-one has not reached the target configuration after stopping at the end configuration. Figures 8, 9 and 10 present the diagrams of the number-two analysed computer test parameters.

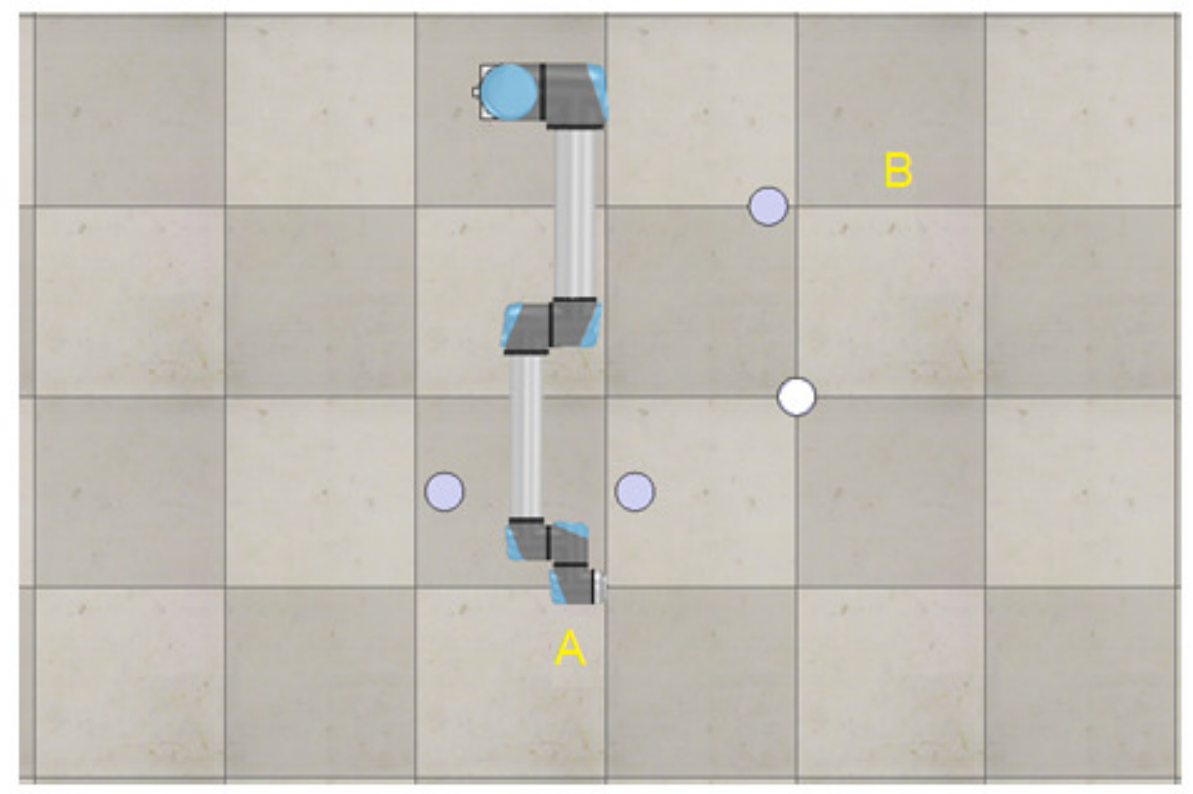

Fig. 7. Results of the test number-two of the intelligent system for planning robot manipulator's real-time movement in an unknown dynamic environment 

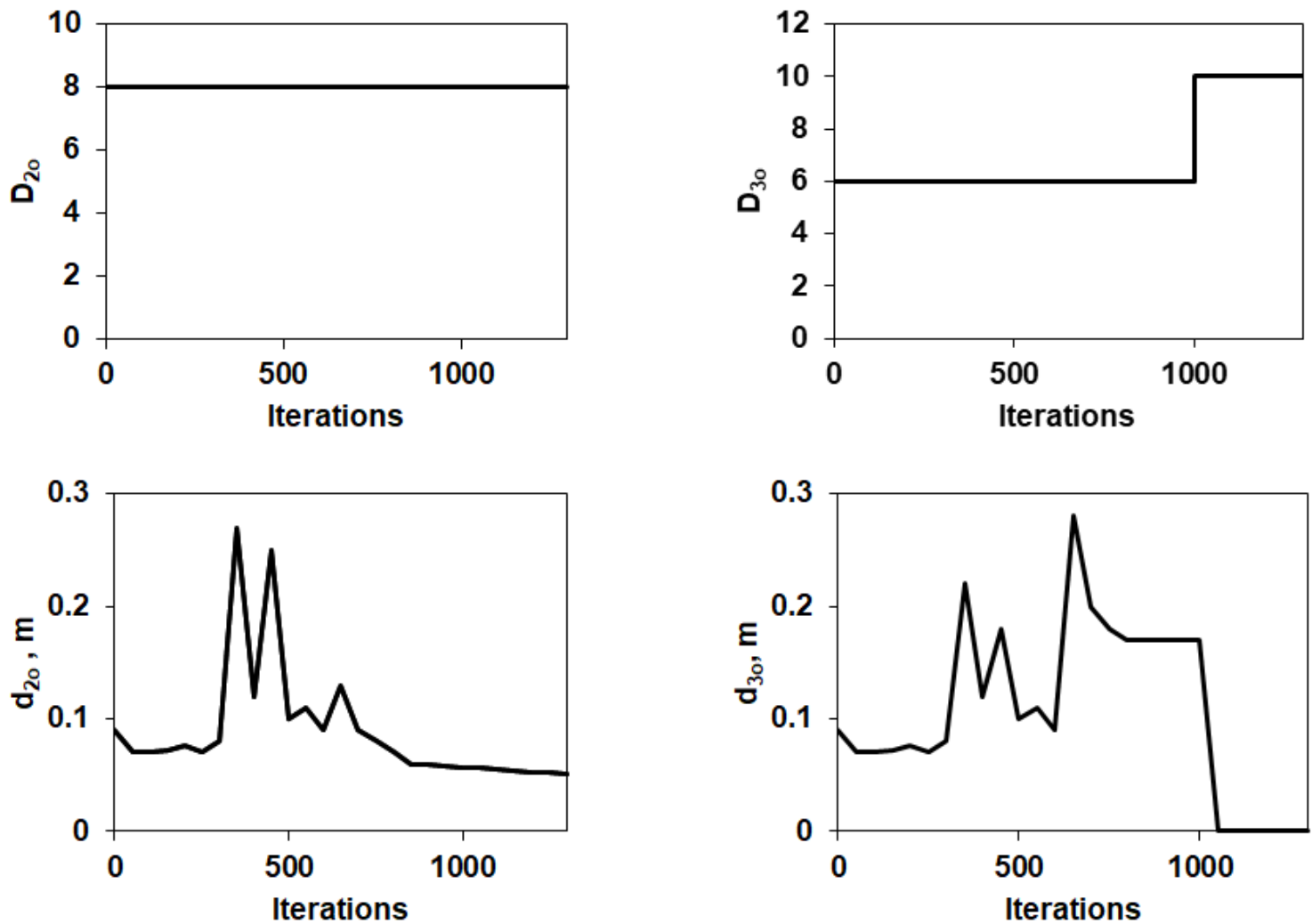

Fig. 8. Test number-two result parameters
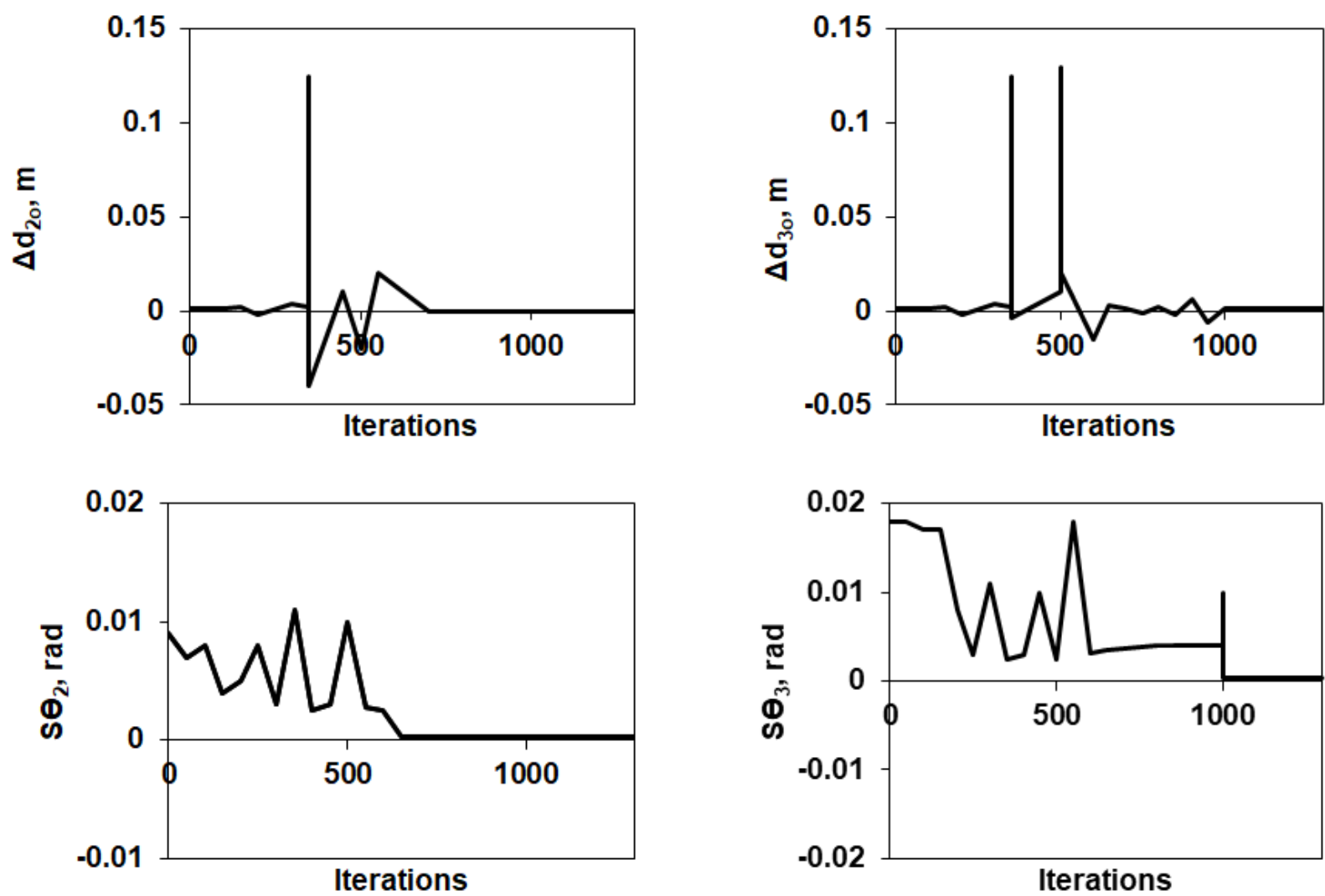

Fig. 9. Test number-two result parameters 

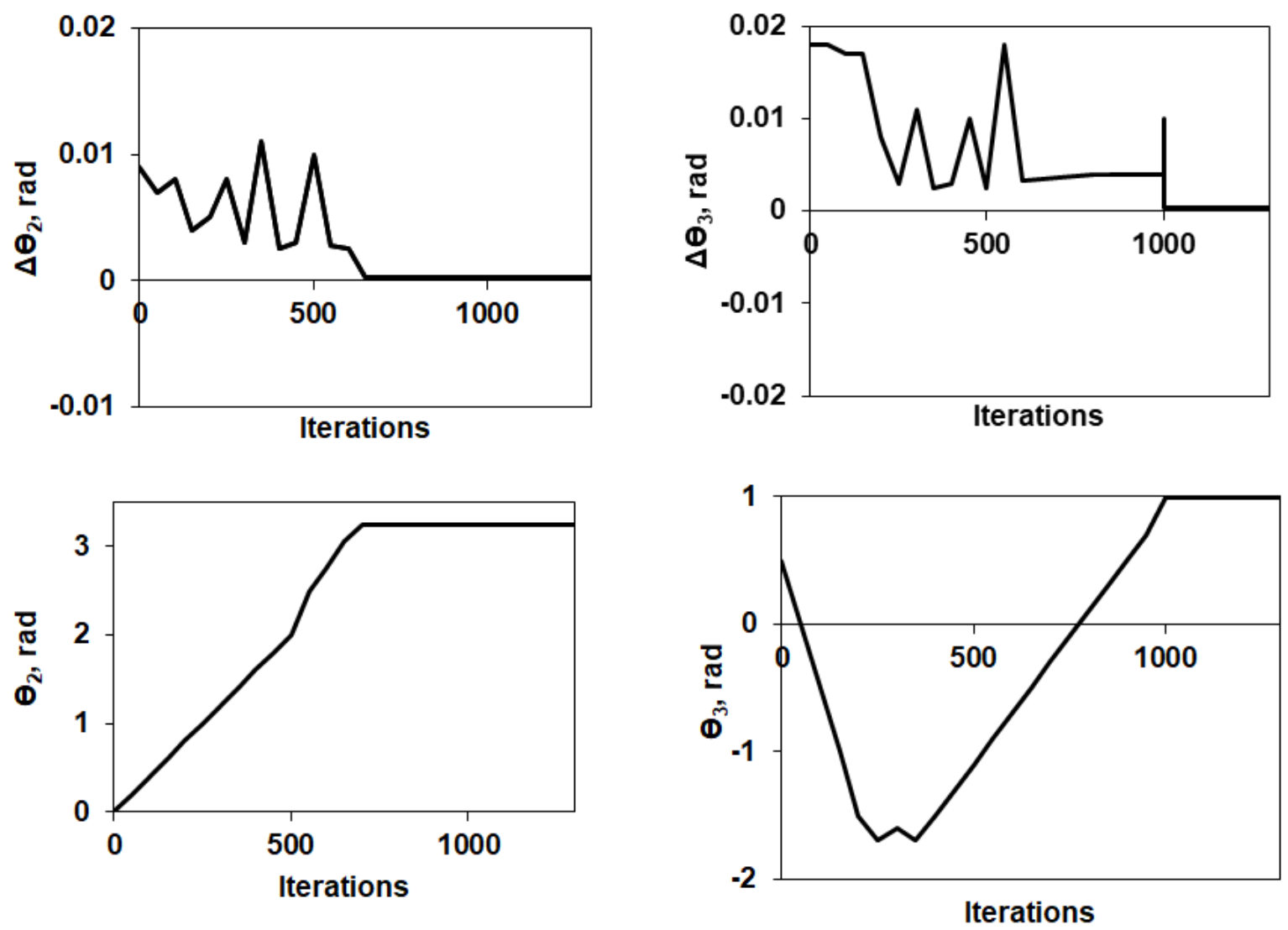

Fig. 10. Test number-two result parameters

\section{Conclusions}

1. The method of designing an intelligent real-time planning system for manipulator's movement in an unknown dynamic environment consisting of four stages is characterized by the change in the structure of the second fuzzy block of each joint resulting from introducing into the system a new parameter - change in the final distance as a result of classification. The use of this parameter allows for calculation of the speed, at which the manipulator and the moving obstacle are approaching one another, which is necessary to avoid the collision. The basis of functioning of the modified fuzzy decision-making mechanism is made up of the designed 108 basic fuzzy rules.

2. In the structures of planning robot-manipulator movement in an unknown static environment, there is no need to use the maximum possible range of step value, therefore the gain coefficients amount to approximately $50 \%$. This range must be ensured in an intelligent planning system, because sometimes the robot is required to respond to changes in the operating zone as quickly as possible. The gain coefficients are, respectivelly, equal to one.

3. During the computer simulation of an intelligent real-time planning system of robot manipulator's movement in an unknown dynamic environment, the largest step of the obstacles moving in the operating zone vertically or horizontally and not moving towards the robot was equal to $0.036 \mathrm{~m}$ in each iteration.

4. In the event when dynamic obstacles move towards the robot manipulator at any speed, and there is no possibility to avoid the collision because the basis of the manipulator is fixed, it is usually suggested to move the robot to a moving platform (mobile robot manipulator).

\section{References}

[1] Dong B., Wang S., Zhou F., Li Y., Wang S., Liu K., Li Y. Critic-Identifier Structure-Based ADP for Decentralized Robust Optimal Control of Modular Robot Manipulators. Proceedings of International conference "Eighth International Conference on Information Science and Technology (ICIST)", June 30 - July 6, 2018, Cordoba, Spain, pp. 21-30. 
[2] Han S., Wang H., Tian Y. Integral backstepping based computed torque control for a 6 DOF arm robot. Proceedings of International conference "29th Chinese Control And Decision Conference (CCDC)", May 28-30, 2017, Chongqing, China, pp. 4055-4060.

[3] Huang A., Liu P. Regressor-free adaptive control of flexible joint robot manipulators with reduced number of estimators. Proceedings of International conference "29th Chinese Control And Decision Conference (CCDC)", May 28-30, 2017, Chongqing, China, pp. 4038-4042.

[4] Zhang D., Wei B. Adaptive control for robotic manipulators. CRC Press, 2016, $425 \mathrm{p}$.

[5] Lee K., Lee J., Woo B., Lee J., Lee Y., Ra S. Modeling and Control of a Articulated Robot Arm with Embedded Joint Actuators. Proceedings of International conference "International Conference on Information and Communication Technology Robotics (ICT-ROBOT)", September 6-8, 2018, Busan, South Korea, pp. 1-4.

[6] Mustafa A., Dhar N.K., Agrawal P., Yerma N.K. Adaptive backstepping sliding mode control based on nonlinear disturbance observer for trajectory tracking of robotic manipulator. Proceedings of International conference. Proceedings of International conference "Control and Robotics Engineering (ICCRE)”, April 1-3, 2017, Bangkok, Thailand, pp. 29-34.

[7] Han X., Ge Z., Zhang K., Wang Z. Design and analysis of a single-input three-DOF parallel manipulator. Proceedings of International conference "2017 IEEE 3rd Information Technology and Mechatronics Engineering Conference (ITOEC)", October 3-5, 2017, Chongqing, China, pp. 324-328.

[8] Liu F., Gao G., Shi L., Lv Y. Kinematic analysis and simulation of a 3-DOF robotic manipulator. Proceedings of International conference " $3 \mathrm{rd}$ International Conference on Computational Intelligence \& Communication Technology (CICT)", February 9-10, 20172017 Ghaziabad, India, pp. $1-5$.

[9] Xiao J., Han W., Wang A. Simulation research of a six degrees of freedom manipulator kinematics based On MATLAB toolbox. Proceedings of International conference "International Conference on Advanced Mechatronic Systems (ICAMechS)", December 6-9, 2017, Xiamen, China, pp. 376-380.

[10] Yagur A.A., Belov A.A. Inverse Kinematics Analysis and Path Planning for 6DOF RSS Parallel Manipulator. Proceedings of International conference "22nd International Conference on System Theory, Control and Computing (ICSTCC)", October 10-12, 2018, Sinaia, Romania, pp. 789-793. 